genetics. Despite the clear importance of the discipline, Ott bemoans what he sees as a historical disregard for the work when, for example, colleagues in the broader genetics community suggest that it is not real genetics but simply a useful tool. Now that a great number of diseases have been mapped and mechanisms described and the approach to this type of study is commonplace, the attention of many geneticists is returning to more fundamental issues, issues for which statistical approaches could become very important, such as the role of 'junk DNA' and large-scale sequence relationships, not to mention solving problems associated with handling and analysing the huge amount of sequence data now being generated around the world.

Real genetics or not, there is nothing trivial about accurately localizing genes responsible for inherited disorders with important repercussions for understanding disease mechanisms, not to mention the immediate ramifications for patient counselling and predictive testing. More often than not, the first clue to unravelling the complexities of many disorders comes with a report linking them to either another disorder or phenotypic trait (as was often the case in the late 1970 s and early 1980 s) or to an anonymous marker sequence. That this approach has become so common and successful is testament to those who pioneered the techniques and refined them to the extent that they are now so accessible. The downside of this is that all too often a linkage study is tacked onto the end of a clinical investigation, with scant attention to the allimportant details of ascertainment bias, parameter choice, treatment of ambiguous phenotypes, pedigree structures, repeat analyses and all the other details that can make or break the analyses. There is also the so-called 'placebo effect'. When considering this problem, which suggests "some spurious linkage results might arise from unrecognized user biases", Ott freely admits that "this is not a logical statement but rather recognizes a human frailty to achieve the most desirable results". Just as many disease genes have been discovered as the result of an accurate linkage study, so too are many projects held up by inadequate linkage studies that have incorrectly focused the community's attention on the wrong part of the genome. To avoid this he advocates separating data collection from the actual linkage calculations so that no individual will be in a position to exert any subtle but important bias.
Ott's frustration may in part explain the vigour with which he sets about teaching statistical genetics and linkage skills. He has, for several years now, run one-week courses for both beginners and advanced students. It is hardly surprising that the courses are oversubscribed and filled by those eagerly working on a plethora of polygenic and complex disorders. Dr Margaret Pericak-Vance of Duke University in Durham, North Carolina, has also launched a residential four-day course covering genetic analysis methods for the medical researcher. She and her coorganizer, Dr Jonathan Haines (Massachusetts General Hospital, Boston) are inundated with requests for help and guidance from those attempting a variety of disease-mapping studies. Despite the varied backgrounds and interests of those approaching Pericak-Vance, she recognized a common thread in that most were trying to dissect the genetic aetiology of complex inherited disorders, an interesting development in a field traditionally concerned with simple monogenic conditions.

The two courses are quite different in approach and style. Ott offers more formal tuition, plenty of hands-on experience with the various linkage programs and specific exercises to illustrate the most important points. In contrast, Pericak-Vance and colleagues have a more relaxed style, with the emphasis on an overall understanding of the various approaches available, continual discussion between students and the large resident faculty and demonstrations using real data. Pericak-Vance points out that the two courses are actually very complementary and recommends that those interested in more technical aspects of the work follow her more general course with Ott's course, which conveniently takes place a few weeks later. Each is likely to continue to be well attended, and as geneticists place more emphasis on the dissection of complex genetic disorders, the sterling efforts of their statistical colleagues are destined to come to the fore in the near future.

\section{Chromosome cleared}

In last month's editorial (Vol. 7, 341-342; 1994), a recessive form of polycystic kidney disease was said to map to chromosome 1 . This should, of course, have read chromosome 6 . We apologize for any confusion this might have caused. 